\title{
Spectrally resolved photon-echo spectroscopy of CdSe quantum dots at far from resonance excitation condition ${ }^{\S}$
}

\author{
DEBABRATA GOSWAMI* (D) \\ Department of Chemistry, Indian Institute of Technology Kanpur, Kanpur, Uttar Pradesh 208 016, India \\ E-mail: dgoswami@iitk.ac.in
}

MS received 12 August 2018; revised 2 September 2018; accepted 4 September 2018; published online 27 September 2018

\begin{abstract}
Spectrally resolved photon echo spectroscopy in the off-resonance condition is reported for the first time to study the coherence and population dynamics of CdSe quantum dots. In this case, the information related to the system dynamics can be inferred indirectly. This is especially useful when such dynamical information might be hidden under the absorption maxima of the sample. We observe that a substantial intensity of the photon echo signal was obtained in two different CdSe quantum dot samples (CdSe 610 and CdSe 640), which have absorption maxima at $620 \mathrm{~nm}$ and $590 \mathrm{~nm}$, respectively. Due to the difference in sizes of these two quantum dots, a small change is observed in the molecular dynamics of these two quantum-dot samples. Specifically, the spectral diffusion of CdSe 640 occurs within the first $50 \mathrm{fs}$, whereas that for CdSe 610 occurs at about $100 \mathrm{fs}$ timescale. The integrated plots of the photon echo signal, as a function of population time, result in two decay constants. The faster among the two decay components is pulse width limited and is in between 30 and $40 \mathrm{fs}$ at different fixed coherence times for both samples. The slower decay component for the $\mathrm{CdSe} 610$ sample is found to be in the range of 75-85 fs, while that for CdSe 640, it is between 82 and $92 \mathrm{fs}$ at different fixed coherence times.
\end{abstract}

Keywords. Free induction decay; spectrally resolved photon echo signal; coherence time; population time; inhomogeneous broadening; femtosecond.

\section{Introduction}

Time-resolved spectroscopic techniques have important applications in studying the evolving structure of complex molecular systems. This is because such evolving structures affect many biological and chemical processes. The molecular dynamics of all the important biological and chemical processes in complex molecular systems range from picoseconds to femtoseconds. ${ }^{1,2}$ Thus, to probe these ultrafast molecular processes experimentally, a sufficiently fast time resolution of the laser system in femtoseconds is needed. The study of molecular structure and dynamics depends on several spectroscopic techniques through absorption, emission, and scattering of light. ${ }^{3,4}$ The utilization of coherent ultrafast femtosecond pulses has resulted in the development of new techniques for probing and modulating molecular reaction dynamics. The transfer of the phase coherence of femtosecond pulses to the

\footnotetext{
*For correspondence

$\S$ Dedicated to Professor M V George on the occasion of his $90^{\text {th }}$ Birth Anniversary.
}

molecular system results in the formation of specific coherent states. The evolution of such states is subsequently recorded to obtain the information at the microscopic level. ${ }^{5-7}$ This principle is applied to obtain detailed and accurate information in the condensed phase. For nonlinear optical effects, the applied electric field should be intense enough to change the optical property of the system. If the applied electric field is weak compared to the internal electric field of the molecule, the optical Polarization 'P' can be expanded perturbatively as a function of the applied radiation field. The induced polarization in the system can be expressed as the sum of linear and nonlinear terms that goes as the power of electric field given by ${ }^{8}$

$\mathbf{P}=P^{(1)}+P^{(2)}+P^{(3)}+P^{(4)}+P^{(5)}+\ldots$

where the term $P^{(1)}$ is linear polarization that is generated by a weak electric field, which controls linear phenomena, such as, absorption, reflection, and refraction. On the hand, the higher order terms in equation (1) represents the nonlinear polarization $\mathrm{P}_{\mathrm{NL}}$. 
$\mathbf{P}_{\mathrm{NL}}=P^{(2)}+P^{(3)}+P^{(4)}+P^{(5)}+\ldots$

For multi-wave mixing experiments, the interaction of several electric fields associated with the wave vector: $\mathrm{k}_{1}, \mathrm{k}_{2}, \mathrm{k}_{3}, \mathrm{k}_{4}, \mathrm{k}_{5}, \ldots \ldots, \mathrm{k}_{\mathrm{n}}$ and the frequency generated: $\omega_{1}, \omega_{2}, \omega_{3}, \omega_{4}, \omega_{5}, \ldots \ldots, \omega_{\mathrm{n}}$ are as per the coherent nonlinear signal associated with the respective wave vectors $\mathrm{k}_{\mathrm{s}}$ and the respective frequency $\omega_{\mathrm{s}}$ as follows:

$\mathrm{k}_{\mathrm{s}}=\mathrm{k}_{1} \pm \mathrm{k}_{2} \pm \mathrm{k}_{3} \pm \mathrm{k}_{4} \pm \mathrm{k}_{5} \ldots \ldots \pm \mathrm{k}_{\mathrm{n}}$

and

$\omega_{\mathrm{s}}=\omega_{1} \pm \omega_{2} \pm \omega_{3} \pm \omega_{4} \pm \omega_{5} \ldots \ldots \pm \omega_{\mathrm{n}}$

The above equation shows that the signal can arise from any combination of the wave vectors or frequency components of interacting electric fields as shown in Figure 1.

For an isotropic medium, the third order polarization is the lowest order of nonlinear polarization induced in the sample. Many third-order nonlinear techniques have been used to study the molecular dynamics of the complex molecular system in liquid and condensed phases. Some of these techniques are Pump Probe, ${ }^{9}$ Hole Burning, ${ }^{10}$ Transient Grating, ${ }^{1}$ and Photon Echo. ${ }^{3}$ Each of these techniques has their characteristic advantage depending on the nature of the system and the specific information required from the experiment. For example, pump-probe and transient-grating experiments are sensitive to population dynamics and provide some indications about dephasing processes. On the other hand, two and three pulse photon echo experiments provide the complete dephasing dynamics of the system in condensed phase. ${ }^{10-14}$

Photon echo is the phenomenon of refocusing the macroscopic polarisation by ultra-short laser pulses. Ultrashort femtosecond pulses with multiple computerinterfaced delays provide control of the coherence and population dynamics and give valuable information about the population relaxation time, inhomogeneous broadening and dephasing time. Photon-echo is

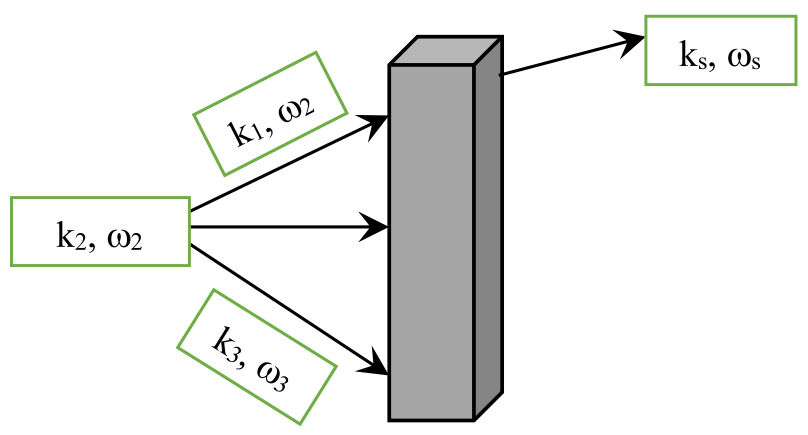

Figure 1. Schematic representation of the different nonlinear processes. attributed to the third order optical nonlinearity of the system.

The elimination of inhomogeneous broadening in the system is important to get pure dynamical information. Many techniques can be used for this purpose, such as Hole Burning. ${ }^{10}$ and two and three pulse Photon Echo, ${ }^{15-19}$ etc. Out of all these techniques, three pulse photon echo technique gives precise results since this technique has two time-delay controls that can easily modulate and manipulate the system, ${ }^{16}$ and hence this is the most reliable technique to study the molecular dynamics in the condensed phase. During the progress of the first time period, the system is in the first coherence state and in the second time period, the system population is either in the ground or the excited electronic state. ${ }^{3}$

In the third evolution period, the system is in the second coherent state. Since the system follows the population state path, either through the ground or the excited state with a controlled time delay, the excited state lifetime is revealed. The spectral diffusion processes that lead to dephasing arises from the fluctuation dynamics, which can be easily obtained without the need for any Markovian separation of timescales. ${ }^{16}$

\subsection{Spectrally resolved three pulse photon echo spectroscopy}

Coherent nonlinear spectroscopy using multiple femtosecond pulses is capable of providing detailed dynamical and spectroscopic information, even in the presence of strong inhomogeneous broadening. Spectrally resolved one-color and two-color three-pulse photon echo (SRPE) spectroscopy in the visible region has been used for probing vibrational and electronic dynamics of simple and complex molecular systems. ${ }^{11,12} \mathrm{~A}$ typical two pulse photon-echo experiment involves passing a series of two laser pulses through the sample (Figure 1). The first pulse, denoted by the wave vector $\mathbf{k}_{\mathbf{1}}$ creates coherence between different energy states of the molecule. This coherence is dephased due to the system-bath interaction and static inhomogeneity. The second pulse, $\mathbf{k}_{\mathbf{2}}$ is applied after a delay time of $\tau$ and is the reason behind the rephasing of the lost coherence. Rephasing regenerates macroscopic polarisation in the specified phase matching direction, and thereby emits an echo pulse. The so-called 'three-pulse photon echo' spectroscopy involves a third laser pulse, in addition to the two pulses stated above. The additional third pulse provides a wider range of dynamics that cannot be probed using the two-pulse photon-echo. In the 'three-pulse photon echo' experiments, the first pulse $\mathbf{k}_{\mathbf{1}}$ creates a coherent superposition of energy states that 
gets gradually lost by dephasing. The second pulse $\mathbf{k}_{\mathbf{2}}$ converts the residual superposition state to either the ground or excited population state. The third pulse, $\mathbf{k}_{\mathbf{3}}$, puts the system back into the second coherent state, which after rephrasing generates the photon echo signal in the $\mathbf{k}_{\mathbf{s}}=-\mathbf{k}_{\mathbf{1}}+\mathbf{k}_{\mathbf{2}}+\mathbf{k}_{\mathbf{3}}$ phase matching direction (Figure 1).

Temporally resolving the photon echo spectra for different excitation wavelengths gives detailed information about the induced nonlinear polarization. ${ }^{20}$ Book and Scherer ${ }^{21}$ used the spectrally resolved stimulated photon echo technique to observe short time dynamics in a dye molecule that are not apparent in time-gated or wavelength integrated photon echo techniques. We have also used the SRPE spectroscopy to study the molecular dynamics of the complex molecular system. ${ }^{22}$

One and two-color three-pulse SRPE spectroscopies for simple and complex molecular systems in the visible region were used for probing vibrational and electronic dynamics in the condensed phase. ${ }^{11}$ The spectrally and temporally resolved photon echo spectra for different excitation wavelengths give detailed information about the induced nonlinear polarisation in the sample. ${ }^{21}$ The spectrally resolved stimulated photon echo technique is used to study the short-term dynamics in a dye molecule that are not apparent in time-gated or wavelength integrated photon echo techniques. ${ }^{21}$ Photon echo technique is a very useful technique for studying the exciton dynamics in GaAs and quantum wells. ${ }^{23,24}$ This technique was also used in the studies of photo-physical dynamics of metalloporphyrins. ${ }^{22}$

Quantum dots shows a great importance in the past few years due to its large application in light-emitting diodes, photovoltaics, laser detectors, bio-labels, and quantum information. ${ }^{25-40}$ In present time CdSe quantum dots and nanorods become an important sample for studying due to its tractable synthesis and size dependent spectroscopic properties. The size dependence property of quantum dots has been characterized by various spectroscopic techniques such as linear absorption, photoluminescence, and fluorescence line narrowing. ${ }^{28,32-38}$ Recently, the study of electronic spectrum and relaxation dynamics of CdSe nanocrystals quantum dots have been done by four-wave mixing experiments such as Transient Absorption, Transient Grating, three-pulse photon echo peak shift (3PEPS), and 2D electronic spectroscopic technique. . $^{2,35,41-55}$

Recently, optical dephasing processes have been used to investigate the simple or complex molecular system. This process involves the solvation dynamics, photophysical processes, single and multiple-exciton migration in quantum dots, photosynthetic antenna,

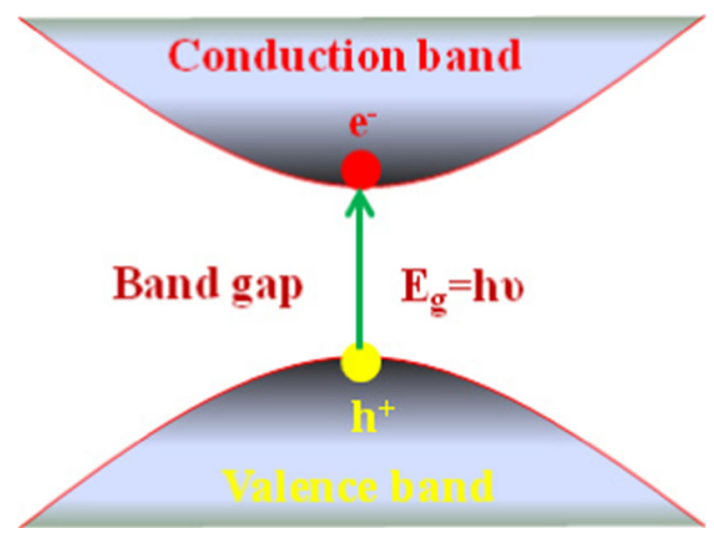

Figure 2. Schematic of valence and conduction band.

and molecular aggregates. ${ }^{1,56-58}$ The advantage of this measurement is its capability to separate the process which leads the homogeneous and inhomogeneous broadening of the spectral transition. ${ }^{59}$ The inhomogeneous broadening is a static effect which is caused by the distribution of the local environment. It can be separated to provide a direct measurement of homogeneous broadening resulting from processes such as molecular collision and system-bath interaction. ${ }^{59}$ Previously CdSe quantum dots have been studies by twopulse photon echo and three-pulse photon echo peak shift as a function of size, temperature, and polarisation. ${ }^{1,56-58,65-70}$

Previous studies have concluded that there are discrete transitions to excitonic states comprising of the hole in the valence band and electron of the conduction band as shown in Figure 2.

CdSe quantum dots have revealed that even the capping ligands situated on their surface play an important role in the dephasing dynamics. ${ }^{64}$ One of the problems in studying the excitonic relaxation and dephasing dynamics of quantum dots is the strong inhomogeneous broadening of transitions. ${ }^{64}$ Such a situation arises because of the finite distribution of shape and size of the dots and also due to their surface defects. Lattice distortions could also contribute to inhomogeneous broadening. ${ }^{64}$ In the previous study of CdSe quantum dots, the quantum beat oscillation was not observed in 3PEPS data due to its fast-dynamical processes. ${ }^{65}$ But SRPE is a very useful technique in overcoming this problem and understanding the ultrafast dynamics of quantum dots. ${ }^{69}$ In this present work, we employ the SRPE in far from resonance condition (towards the red edge of the spectrum) to unravel information on $\mathrm{CdSe}$ quantum dots. 


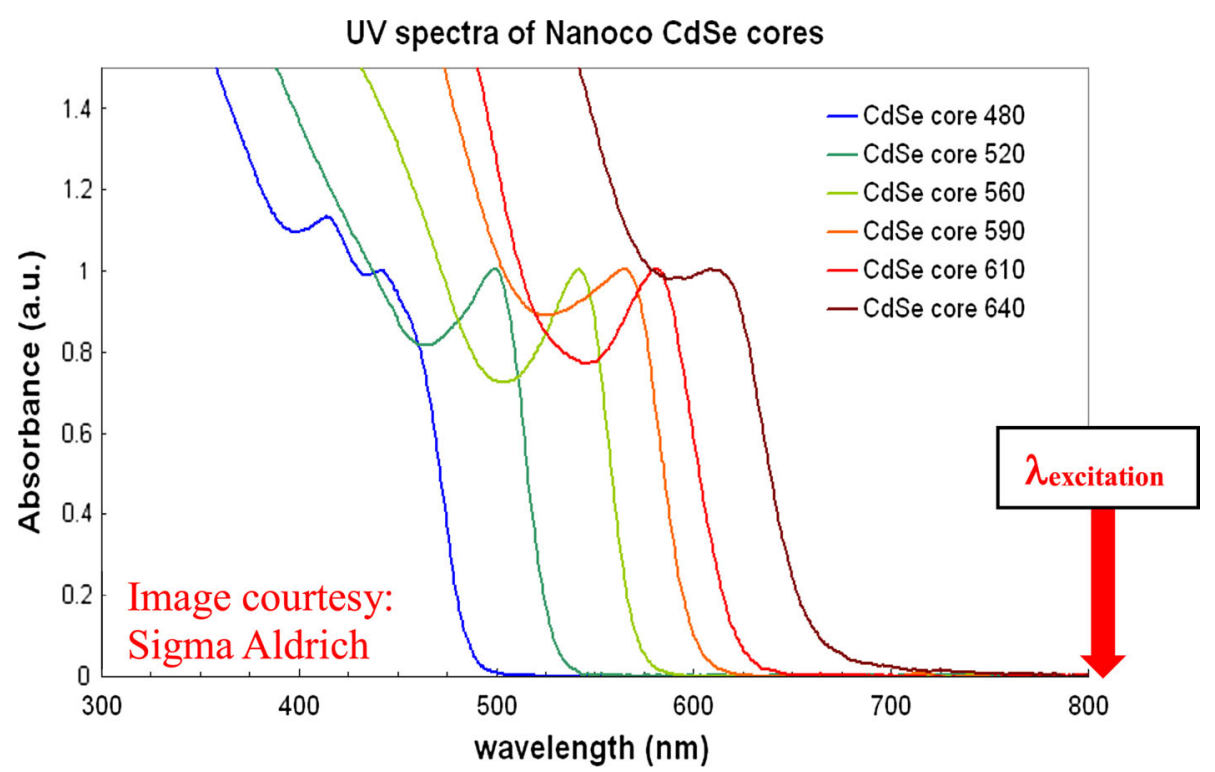

Figure 3. Absorption spectra of various CdSe quantum dots sample in toluene. The marker arrow shows the excitation laser wavelength for SRPE experiments.

\section{Experimental and theoretical}

\subsection{Chemicals and sample preparation}

Commercially available Lumidot CdSe quantum dots of various sizes: CdSe 560, CdSe 590, CdSe 610 and CdSe 640 were procured from Sigma Aldrich Company. The numbers next to CdSe indicate the characteristic emission wavelength, which in turn is determined by the dot size. Figure 3 shows the corresponding absorption spectra for all our CdSe quantum dots.

The dots are surface-stabilized by organic ligands and are dispersed in toluene. For sample preparation, $1 \mathrm{~mL}$ of the quantum dot (dispersed in toluene of $5 \mathrm{mg} / \mathrm{mL}$ concentration) was taken and diluted to $10 \mathrm{~mL}$, to prepare the stock solution. It was successively diluted to $25 \mathrm{~mL}$ to make the final concentration of $0.2 \mathrm{mg} / \mathrm{mL}$ and then introduced into a flow cell.

\subsection{Laser system and experimental setup}

The 82-MHz Ti:Sapphire oscillator (MaiTai, Spectra-Physics) operating at the center wavelength of $810 \mathrm{~nm}$ (bandwidth 60 $\mathrm{nm}$ ) and pulse-width of $25 \mathrm{fs}$ is used as a seed. This seed pulse is amplified through a commercial Ti:Sapphire Regenerative Amplifier (Spitfire XP, Spectra-Physics) that is being pumped by the $527 \mathrm{~nm}$ doubled Nd:YLF laser (Empower, Spectra-Physics) operating at $1 \mathrm{kHz}$ repetition rate. We used the resulting amplified pulses of 30 fs duration, which was centered at $810 \mathrm{~nm}$. Though the amplified laser pulse repetition rate is tunable, we fixed it at $1 \mathrm{kHz}$ for our experiment. Figure 4 shows the schematic diagram of the experimental setup for SRPE spectroscopy.
The ultrafast laser beam from the amplifier was split into three beams of equal intensity by using appropriate thin ultrafast beam splitters (BS 70:30 and 50:50) and sent through variable delay lines consisting of retroreflectors and motorized translations stages (Newport, UTM-CC-0.1). The pulses were arranged in boxcar geometry as shown in Figure 4 in a way so that each beam was $1 \mathrm{~cm}$ apart from each other. These laser beams were focused into the flowing sample by using the $20 \mathrm{~cm}$ achromatic lens. The sample was flown in a $0.2 \mathrm{~mm}$ flow cell by using the peristaltic pump consisting of Teflon tube to avoid the burning and photodegradation of sample. The photon echo signal is generated at the fourth corner of the boxcar square after the macroscopic dipole moment is rephased (in the direction of $\mathbf{k}_{\mathbf{s}}$ ). The generated photon-echo signal is collected through a miniature grating spectrometer (HR-2000, Ocean Optics). The data was recorded on the computer by using the LabVIEW program.

\subsection{Theoretical background}

Photon echo spectroscopy techniques are generally used to probe molecular dynamics of the complex molecular system in which absorption bands are very congested. ${ }^{11,12}$ Photon echo spectroscopy is a four-wave mixing technique in which the multiple laser pulses in the appropriate geometry induces nonlinear polarisation in the sample. The measurement of nonlinear polarisation induced in the sample gives the information about the molecular dynamics of the complex molecular system. ${ }^{11}$ In our particular experiment, we used boxcar geometry, where four pulsed laser beams of nearly equal intensity propagate parallel to each other as have been shown in Figure 4. The three laser pulses associated with wave-vectors: $\mathbf{k}_{\mathbf{1}}, \mathbf{k}_{\mathbf{2}}$, and $\mathbf{k}_{\mathbf{3}}$ are focused on flowing sample by using an achromatic lens (as shown schematically in 


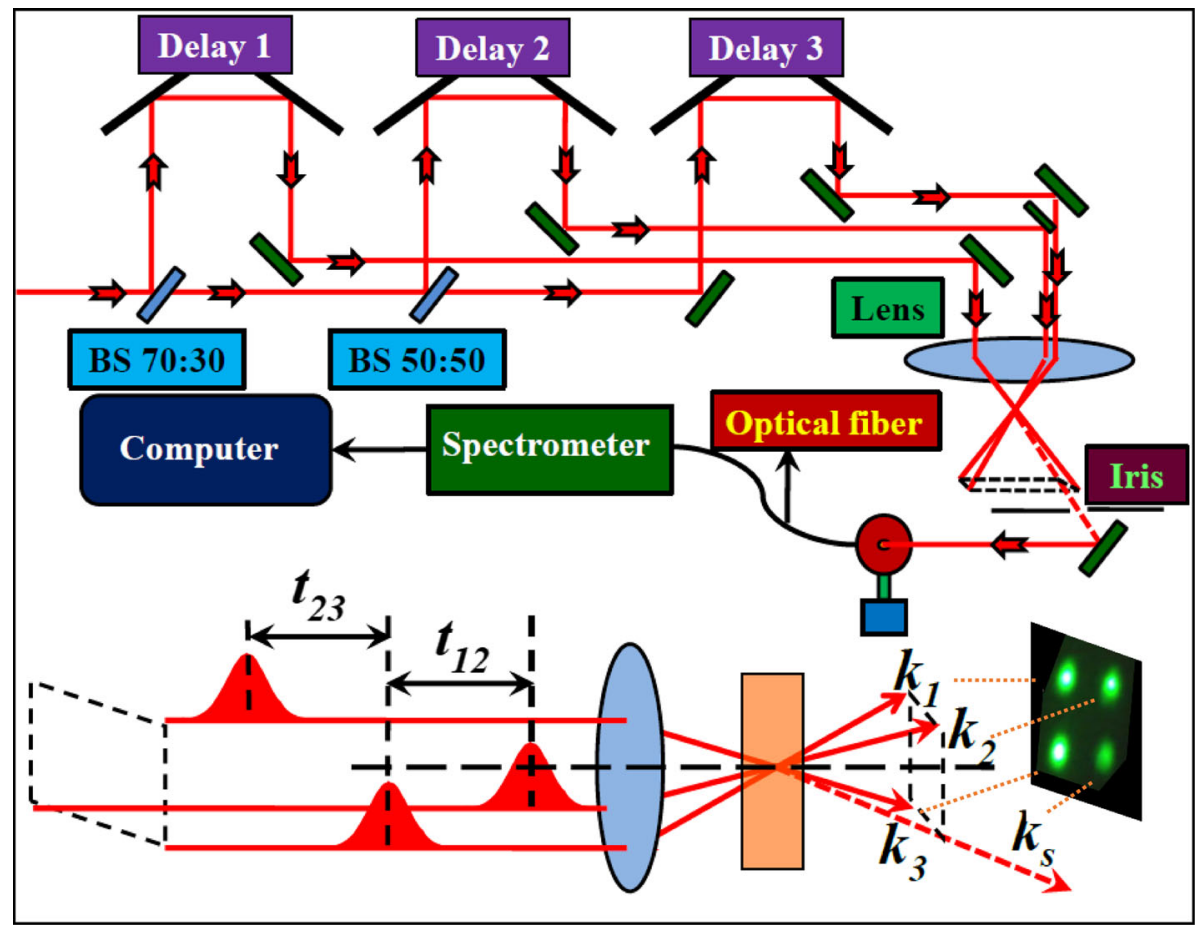

Figure 4. Schematic of the experimental setup and pulse sequence for three pulse photon echo technique. Image of the different pulses and the echo signal is connected with dotted lines for clarity.

Figure 1). The resulting photon echo signal is at the fourth corner of the square after rephasing of macroscopic dipole moment. The time duration between the first two pulses is called the coherence time $\left(\mathrm{t}_{12}\right)$ while the time duration between the second and third pulse is called population time $\left(t_{23}\right)$. The total electric field $\mathbf{E}(\mathbf{t})$ applied in our four-wave mixing experiment is given by: ${ }^{71}$

$$
\begin{aligned}
E(t)= & E_{1}\left(t+t_{12}+t_{23}\right) e^{-i\left(\omega_{1} t-k_{1} . r\right)} \\
& +E_{2}\left(t+t_{23}\right) e^{-i\left(\omega_{2} t-k_{2} . r\right)} \\
& +E_{3}(t) e^{-i\left(\omega_{3} t-k_{3} . r\right)}
\end{aligned}
$$

where $\mathbf{E}_{\mathbf{i}}(\mathbf{t}), \boldsymbol{\omega}_{\mathbf{i}}$ and $\mathbf{k}_{\mathbf{i}}$ represents electric field, central laser frequency and the wave vector of the $i^{\text {th }}$ incident electric field. By the interaction of three laser pulses, the third order nonlinear polarisation $\left(\mathrm{P}^{(3)}\right)$ induced in the sample is given by: ${ }^{71}$

$$
\begin{aligned}
P^{(3)}= & \int_{0}^{\infty} d t_{3} \int_{0}^{\infty} d t_{2} \int_{0}^{\infty} d t_{1} E_{1}^{*}\left(t+t_{12}+t_{23}-t_{3}-t_{2}-t_{1}\right) \\
& \times E_{2}\left(t+t_{23}-t_{3}-t_{2}\right) \times E_{3}\left(t-t_{3}\right) \\
& \times R^{(3)}\left(t_{3}, t_{2}, t_{1}\right)
\end{aligned}
$$

where $\mathrm{R}^{(3)}$ is a third order nonlinear response function. The above nonlinear response function $\mathrm{R}^{(3)}$ is the linear combination of both rephasing $\left(R_{a}\right)$ and non-rephasing $\left(R_{b}\right)$ response to the macroscopic dipole moment. Thus, the total response function is: ${ }^{72}$

$R^{(3)}\left(t_{1}, t_{2}, t_{3}\right)=R_{a}\left(t_{1}, t_{2}, t_{3}\right)+R_{b}\left(t_{1}, t_{2}, t_{3}\right)$
Here, the rephasing response function $\left(\mathrm{R}_{\mathrm{a}}\right)$ contributes as a photon-echo-like-signal in the third-order nonlinear polarization. The mathematical expression for rephasing response function is given by: ${ }^{72}$

$$
\begin{aligned}
& R_{a}\left(t_{3}, t_{2}, t_{1}\right)=\left[R_{2}\left(t_{3}, t_{2}, t_{1}\right)+R_{3}\left(t_{3}, t_{2}, t_{1}\right)\right] \\
& \quad \times E_{3}\left(t-t_{23}-t_{3}\right) \exp \left[-i \omega_{3}\left(t-t_{23}-t_{3}\right)\right] \\
& \quad \times E_{2}\left(t-t_{3}-t_{2}\right) \exp \left[-i \omega_{2}\left(t-t_{3}-t_{2}\right)\right] \\
& \quad \times E_{1}^{*}\left(t+t_{12}-t_{3}-t_{2}-t_{1}\right) \\
& \quad \times \exp \left[i \omega_{1}\left(t+t_{12}-t_{3}-t_{2}-t_{1}\right)\right]
\end{aligned}
$$

where $\mathrm{R}_{2}$ and $\mathrm{R}_{3}$ are third-order nonlinear response functions. The non-rephasing response function $\left(\mathrm{R}_{\mathrm{b}}\right)$ contributes as FID like signal in the in the third order nonlinear polarisation. The mathematical expression for non-rephasing response function is given by: ${ }^{72}$

$$
\begin{aligned}
& R_{b}\left(t_{3}, t_{2}, t_{1}\right)=\left[R_{1}\left(t_{3}, t_{2}, t_{1}\right)+R_{4}\left(t_{3}, t_{2}, t_{1}\right)\right] \\
& \quad \times E_{3}\left(t-t_{23}-t_{3}\right) \exp \left[-i \omega_{3}\left(t-t_{23}-t_{3}\right)\right] \\
& \times E_{2}\left(t-t_{3}-t_{2}-t_{1}\right) \exp \left[-i \omega_{2}\left(t-t_{3}-t_{2}+t_{1}\right)\right] \\
& \times E_{1}^{*}\left(t-t_{12}-t_{3}-t_{2}\right) \exp \left[i \omega_{1}\left(t-t_{12}-t_{3}-t_{2}\right)\right]
\end{aligned}
$$

where $\mathrm{R}_{1}$ and $\mathrm{R}_{4}$ are third-order nonlinear response function.

The Feynman diagram given in Figure 5 is an important tool to explain our experimental observation. The interaction of three laser pulses in proper phase matching direction is given Feynman diagram. ${ }^{72}$ The two vertical lines in Figure 5 
for $\mathrm{R}_{1}, \mathrm{R}_{2}, \mathrm{R}_{3}$ and $\mathrm{R}_{4}$ response functions represents the time evolution of the ket and the bra of the density matrix.

The time increase corresponds to going from the bottom to top in the Feynman diagram. Every arrow is representing the interaction of pulses with the system. An arrow pointing towards the vertical line for both bra and ket side of the density matrix represents the absorption while the arrows pointing away from the vertical line represents the emission. For each response function diagram has a sign $(-1)^{n}$, where $n$ is the number of interactions from the bra side of the Feynman diagram. The last arrow represents the generated third-order nonlinear signal. For inhomogeneously broadening ensembles, the Gaussian distribution for a simple two-level system is given by: ${ }^{11}$

$G(\omega)=\exp \left[-\frac{\left(\omega-\omega_{12}\right)^{2}}{\Gamma^{2}}\right]$

where $\Gamma$ is $1 / \mathrm{e}$ half-width. Now the final expression for third-order nonlinear response function including the contribution of inhomogeneously broadening ensemble average is given by: ${ }^{72}$

$$
\begin{aligned}
R_{1}\left(t_{3}, t_{2}, t_{1}\right)= & \exp \left[-\mathrm{i} \omega_{12}\left(t_{3}+t_{1}\right)\right] \exp \left[-\Upsilon_{12}\left(t_{3}+t_{1}\right)\right] \\
& \left.\times \exp \left[-\Upsilon_{12} t_{2}\right] \exp \left[-\Gamma^{2}\left(t_{3}+t_{1}\right)^{2} / 4\right)\right] \\
= & R_{4}\left(t_{3}, t_{2}, t_{1}\right) \\
R_{2}\left(t_{3}, t_{2}, t_{1}\right)= & \exp \left[-i \omega_{12}\left(t_{3}-t_{1}\right)\right] \exp \left[-\Upsilon_{12}\left(t_{3}+t_{1}\right)\right] \\
& \left.\times \exp \left[-\Upsilon_{12} t_{2}\right] \exp \left[-\Gamma^{2}\left(t_{3}-t_{1}\right)^{2} / 4\right)\right] \\
= & R_{3}\left(t_{3}, t_{2}, t_{1}\right)
\end{aligned}
$$

where $\omega_{12}$ is the transition frequency, $\gamma_{12}=1 / T_{2}$ and $\gamma_{1}=$ $1 / T_{1}$. The $T_{1}$ excited state lifetime and $T_{2}$ is the dephasing time of the transition.

The third order nonlinear polarisation for spectrally resolved photon echo signal (SRPE) is determined by the Fourier transformation of time domain $3^{\text {rd }}$ order polarisation given in equation (5). Thus, the frequency domain third-order nonlinear polarisation is given by: ${ }^{11-14,21,73,74}$

$\dot{P}^{(3)}\left(\omega, t_{12}, t_{23}\right)=\int_{-\infty}^{\infty} P^{(3)}\left(t, t_{12}, t_{23}\right) e^{i \omega t} d t$

Now the third or nonlinear polarisation including response functions given in equations (7a), (7b), (9a) and (9b) assuming delta function for two-level systems is given by: ${ }^{72}$

$$
\begin{aligned}
& \dot{\mathrm{P}}^{(3)}\left(\omega, t_{12}, t_{23}\right) \propto N \mu_{12}^{4} \exp \left[\mathbf{i} \omega_{3} t_{23}\right] \exp \left[-\Upsilon_{1} t_{23}\right] \\
& \operatorname{Xexp}\left[\left(\frac{\left.i\left(\omega_{12}-\omega\right)+\Upsilon_{12}\right)^{2}}{\Gamma^{2}}\right)\right] X\left\{\exp \left[-\mathbf{i} \omega_{12}\left(t_{23}-t_{12}\right)\right]\right. \\
& \operatorname{erfc}\left[\frac{i\left(\omega_{12}-\omega\right)}{\Gamma}+\frac{\Upsilon_{12}}{\Gamma}+\frac{t_{12} \Gamma}{2}\right] \Theta\left(t_{12}\right) \Theta\left(t_{23}\right) \\
& +\exp \left[-i \omega_{12}\left(t_{23}+t_{12}\right)\right] \operatorname{Xexp}\left[-2 \Upsilon_{12} t_{12}\right] \\
& \left.\operatorname{erfc}\left[\frac{i\left(\omega_{12}-\omega\right)}{\Gamma}+\frac{\Upsilon_{12}}{\Gamma}-\frac{t_{12} \Gamma}{2}\right] \Theta\left(-t_{12}\right) \Theta\left(t_{23}\right)\right\}
\end{aligned}
$$

where $\Theta$ is the Heaviside step function. The signal intensity for spectrally resolved (time-integrated) photon echo signal is given by: ${ }^{25}$
$S\left(\lambda_{d}, t_{12}, t_{23}\right) \propto \int_{0}^{\infty}\left|\hat{\mathrm{P}}^{(3)}\left(\lambda_{\mathrm{d}}, \mathbf{t}_{12}, \mathbf{t}_{23}\right)\right|^{2} \mathrm{dt}$

where $\lambda_{d}$ is the wavelength detected by the spectrometer. At zero coherence time $\left(\mathrm{t}_{12}\right)$ when two laser pulses overlap the signal spectrum includes the contribution of population grating. So, the final expression may then be returned as ${ }^{13}$ :

$$
\begin{aligned}
S_{D}\left(\lambda_{d}, t_{12}, t_{23}\right)= & S_{S R P E}\left(\lambda_{d}, t_{12}, t_{23}\right) \\
& +\eta\left(\lambda_{d}, t_{12}, t_{23}\right) I_{P R}\left(\lambda_{d}\right)
\end{aligned}
$$

where $\boldsymbol{\eta}\left(\boldsymbol{\lambda}_{\boldsymbol{d}}, \boldsymbol{t}_{\mathbf{1 2}}, \boldsymbol{t}_{\mathbf{2 3}}\right)$ is the efficiency of the population grating and $\mathbf{I}_{\mathbf{P R}}\left(\boldsymbol{\lambda}_{\mathbf{d}}\right)$ is the spectral density of the probe pulse which is detected by the spectrometer as a function of the central wavelength of the laser pulses. The efficiency of population grating $(\boldsymbol{\eta})$ is proportional to the $\left[\exp \left(-t_{23} / \nu_{\text {life }}\right)-\right.$ $\left.\exp -\left(\mathrm{t}_{23} / \nu_{\text {rise }}\right)\right]$, where $\nu_{\text {life }}$ and $\nu_{\text {rise }}$ are the lifetime and the formation time of the population grating, respectively.

\section{Results and Discussion}

\subsection{Photon echo spectroscopy of CdSe quantum dots}

Numerous one color three-pulse photon echo studies of a variety of compounds have been reported in the literature, ranging from dyes, biological molecules, semiconductors, quantum dots, etc. $22,41-55$ Typical photon echo experiments are done in the resonance condition, i.e., when the central wavelength of the laser pulse is around the region of maximum absorption. When the first two pulses fall on the sample, it gets excited, and a population grating is formed. The efficiency of the grating is likely to be dependent on the extent of absorption. That is the reason as to why three-pulse photon-echo peak shift experiments are generally carried out with the laser wavelength close to that for maximum absorption. ${ }^{61-63}$ However, the strong inhomogeneous broadening for resonant transitions of quantum dots lead to ultrafast excitonic relaxation and dephasing dynamics, which makes such studies difficult. ${ }^{65}$ In our experiment, we try to see whether we can perform photon echo experiments in the far from resonance condition as well. A laser pulse of central wavelength $810 \mathrm{~nm}$ is used in our SRPE experiments. From Figure 3 it is clear that the absorbance at $810 \mathrm{~nm}$ is quite negligible as compared to the maximum absorption. But there is still some finite absorption at this wavelength. Due to this, the photon echo signal of substantial intensity is observed at $810 \mathrm{~nm}$ which is far from the resonance condition or towards the reddish edge of the absorption spectrum in CdSe 610 and CdSe 640 quantum dots. Though the overall signal at such off-resonance condition is lower than at the resonance wavelengths, the ultrafast pulse-widths in our experiments provide the necessary advantage to monitor ultrafast relaxation dynamics. The 

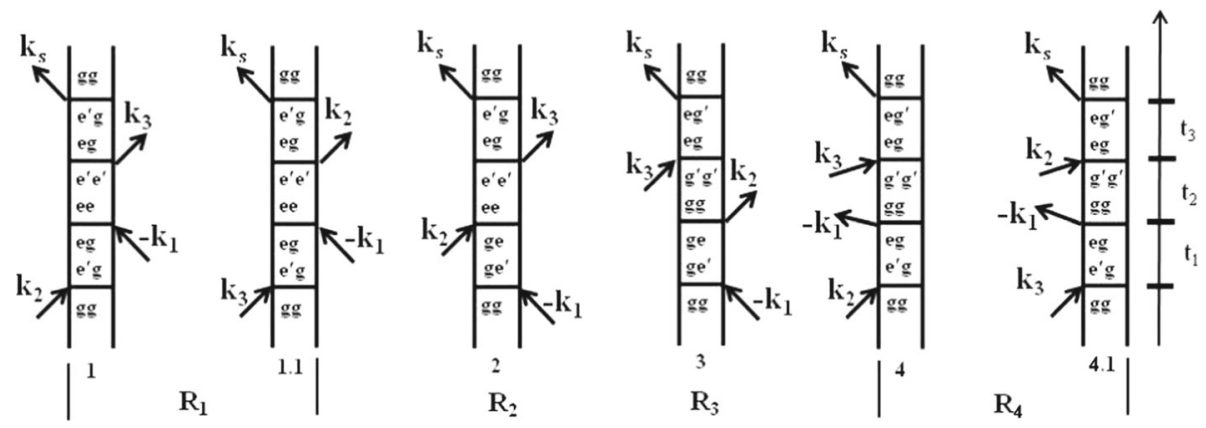

Figure 5. Double-sided Feynman diagram.

valence and conduction band for CdSe quantum dots exhibit different states near the band edge. ${ }^{75}$ These states allow the respective charge carrier to undergo different relaxation via coupling to ligands, acoustic, and optical phonon mode. ${ }^{27,45,75-81}$ In a semiconductor nanocrystal, the electron-hole correlated processes play an important role in electronic relaxation. The electron relaxation occurs due to the sparer density of states for electrons near the band edge or by angular momentum consideration. In quantum dots, the electron is relaxed via an Anger-like mechanism, in which electron wave function overlap allows the electron to transition while simultaneously exciting a hole in the valence band. The above process occurs due to fast band-edge relaxation for $\mathrm{CdSe}$ quantum dot states. ${ }^{25,71,77,82}$

Salvador et al., assumed the two-level systems for performing the simulation for 3PEPS data of CdSe quantum dots. ${ }^{60}$ For our experimental analysis, we are also assuming two-level systems for $\mathrm{CdSe}$ quantum dots. For studying the excitonic dynamics and phonon coupling, we are applying optical response theory used for fourwave mixing experiment. In our experiment, photon echo signal was spectrally resolved in a spectrometer, and the data was collected by scanning the coherence time for various fixed population time and also by scanning the population time for various fixed coherence times. Figure 6(A-D) shows the spectrally resolved photon echo plots by scanning the coherence time $\left(\mathrm{t}_{12}\right)$ at fixed population time $\left(\mathrm{t}_{23}\right)$ times of $0,50,100$ and 150 $\mathrm{fs}$, for CdSe 610 in toluene.

At zero coherence time, the spectrally resolved contour plot shows a tilted profile, which implies blueshifting of the photon echo signal during positive coherence time and red shifting of the photon echo spectrum during negative population times. This indicates the inhomogeneous broadening in the spectrum caused by the different properties induced by the fluctuation in size, shape, and symmetry of each nanocrystal in quantum dots ensembles. ${ }^{60}$ The main cause of inhomogeneous broadening in the spectrum is sample size distribution. There are some oscillations observed in the negative direction of coherence time due to the vibrational modes. Generally, for semiconductor nanocrystal, the exciton is coupled to Longitudinal Optical phonons due to polar Fröhlich coupling. In this coupling, the optically excited electronic charge distribution coupled via Coulomb interaction to the charge density induced by the vibrational motion of the lattice atoms. There is negligible wavelength shift observed when we increase the fixed population time from 0 to $100 \mathrm{fs}$ time scale that indicates the population relaxation dynamics. In Figure 6(B), at 50 fs fixed population time, the shape of the contour plots starts becoming symmetric with respect to zero coherence time and the signal maxima shifted towards the zero-coherence time $\left(t_{12}\right)$. For negative coherence, the FID contribution is more in the spectra according to the Feynman diagram in Figure 5. It is seen in Figure 6(A-C), that the FID contribution is more in the spectra that indicate inhomogeneous broadening in the spectra. The inhomogeneous state may be present for more than $100 \mathrm{fs}$ population time, but it is not observable in our experiment because our signal intensity almost decayed at $150 \mathrm{fs}$ timescale.

For $\mathrm{CdSe} 640$, the same excitation pulsed laser source with a center wavelength of $810 \mathrm{~nm}$ was used, and the results are shown in Figure 7. The spectrally resolved photon echo signal at zero population time at fixed zero coherence time was centered at $810 \mathrm{~nm}$, and a blue shift was observed for positive coherence times and redshift for negative coherence times that indicates the inhomogeneous broadening in the spectra as shown in Figure 7(A). In Figure 7(B) at 50 fs fixed population time $\left(t_{23}\right)$, the shape of spectra becomes symmetrical and the signal intensity is centered near zero fs coherence time. There is no wavelength shift observed as we increase the fixed population time from 0 to $50 \mathrm{fs}$, which indicates that spectral diffusion process occurs at $\sim 50$ fs timescale for CdSe 640 quantum dot.

We have also studied the time-resolved photon echo spectroscopy for both CdSe 610 and CdSe 640 quantum 

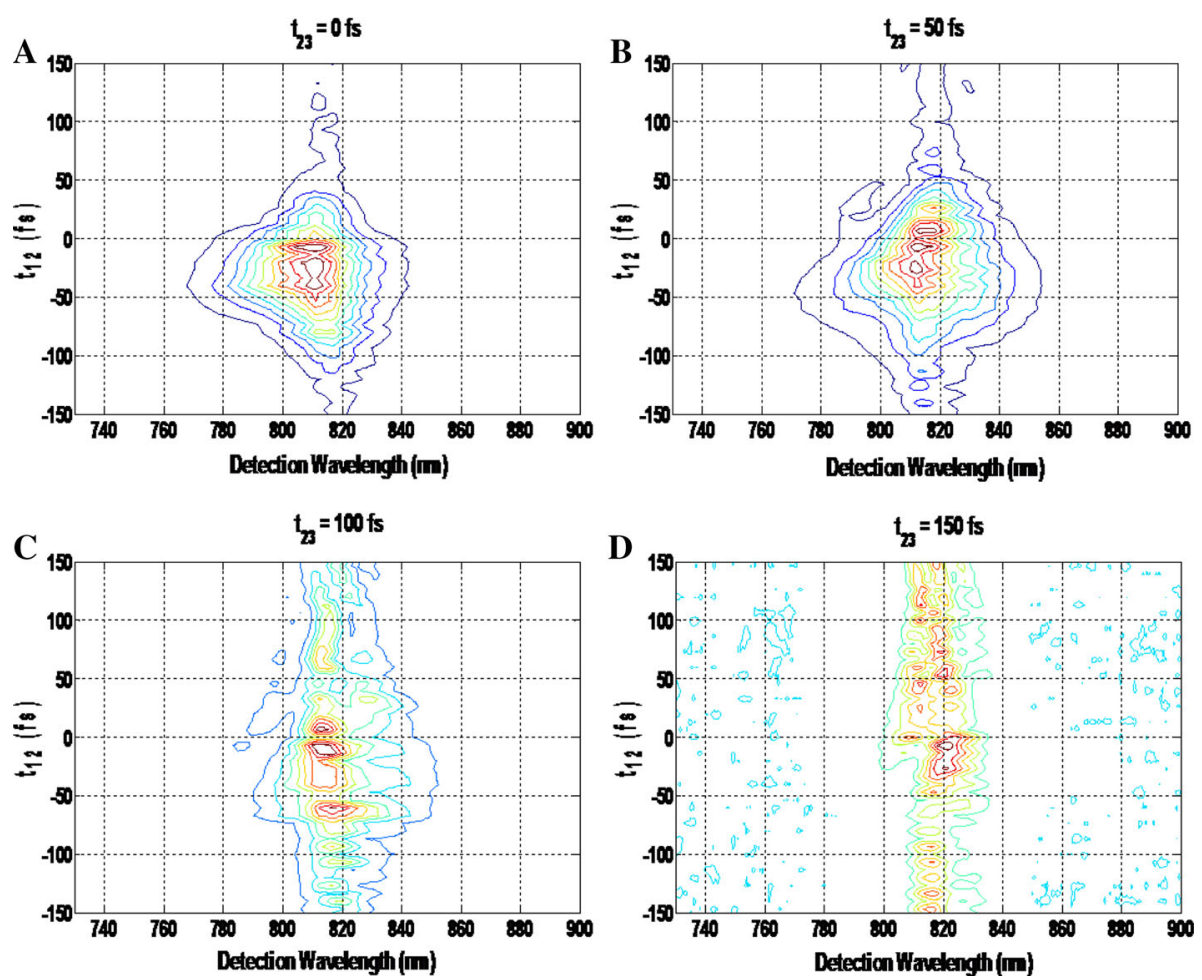

Figure 6. Spectrally resolved photon echo plots for CdSe 610 in toluene taken while scanning the coherence time for various fixed population times $\left(\mathrm{t}_{23}\right)$ of $(\mathrm{A})$ 0fs, (B) 50fs, (C)100fs, (D) 150fs.
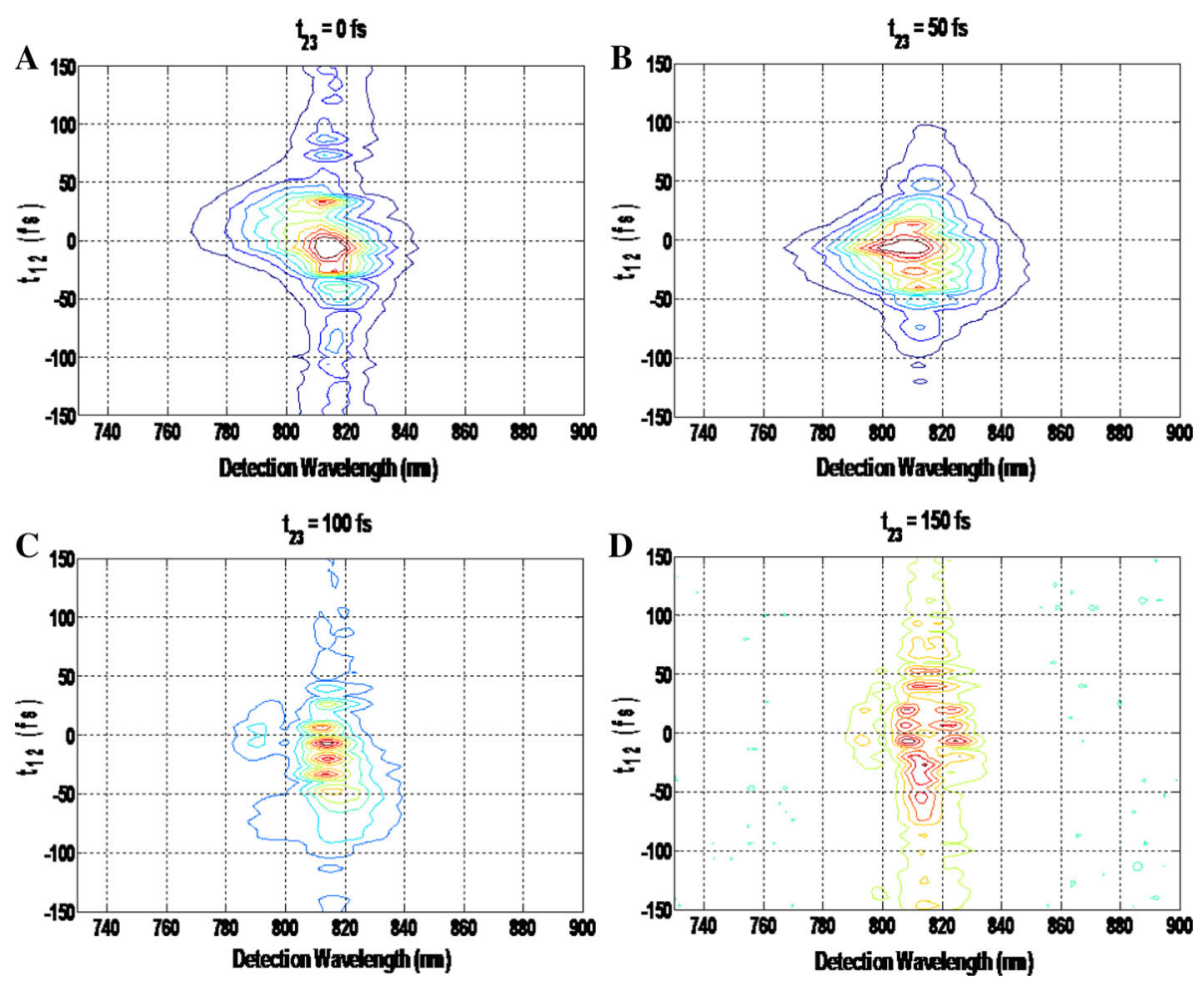

Figure 7. Spectrally resolved photon echo plots for CdSe 640 in toluene taken while scanning the coherence time $\left(\mathrm{t}_{12}\right)$ for various fixed population times $\left(\mathrm{t}_{23}\right)$ of $(\mathrm{A})$ 0fs, (B) $50 \mathrm{fs},(\mathrm{C}) 100 \mathrm{fs},(\mathrm{D}) 150 \mathrm{fs}$. 

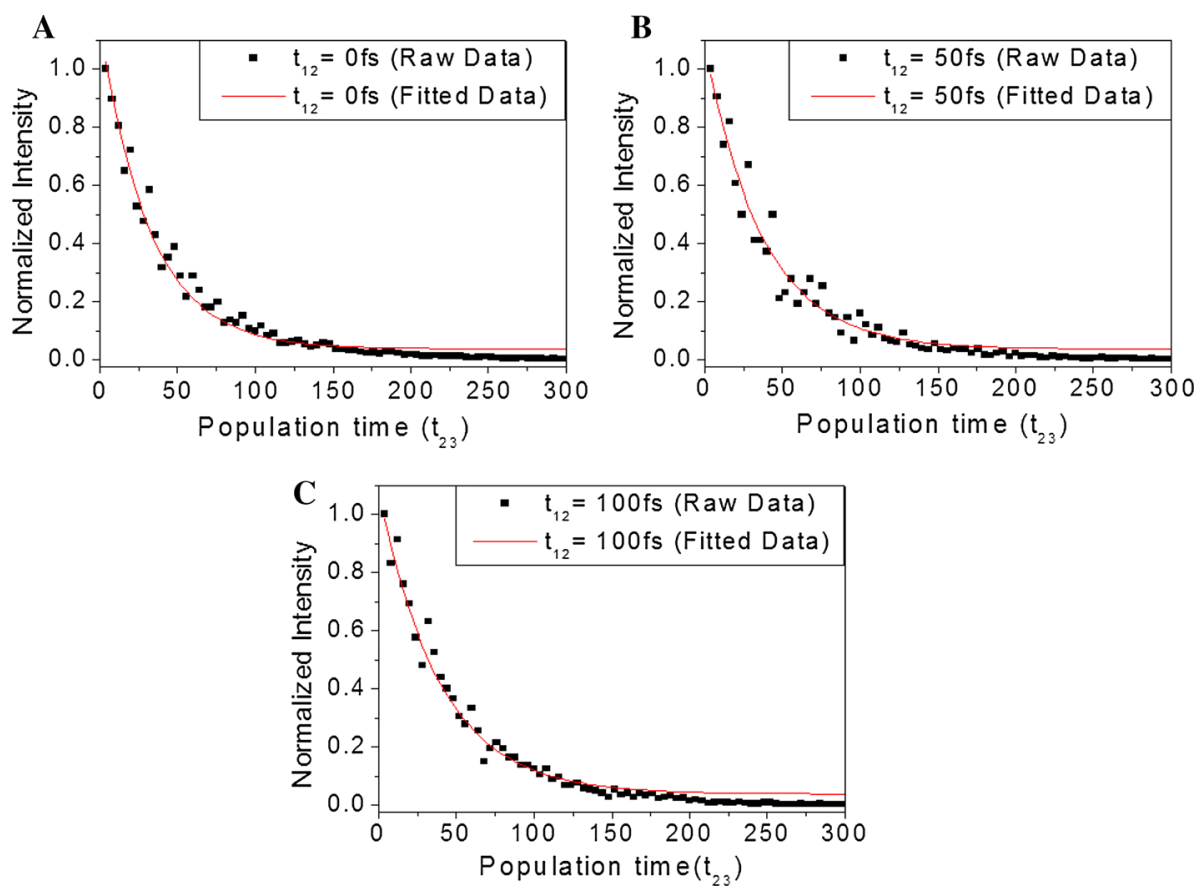

Figure 8. Fitted integrated plots of photon echo signal for CdSe 610 quantum dot in toluene solvent at four (A to D) specific coherence times $\left(\mathrm{t}_{12}\right)$ while varying the population $\left(\mathrm{t}_{23}\right)$.
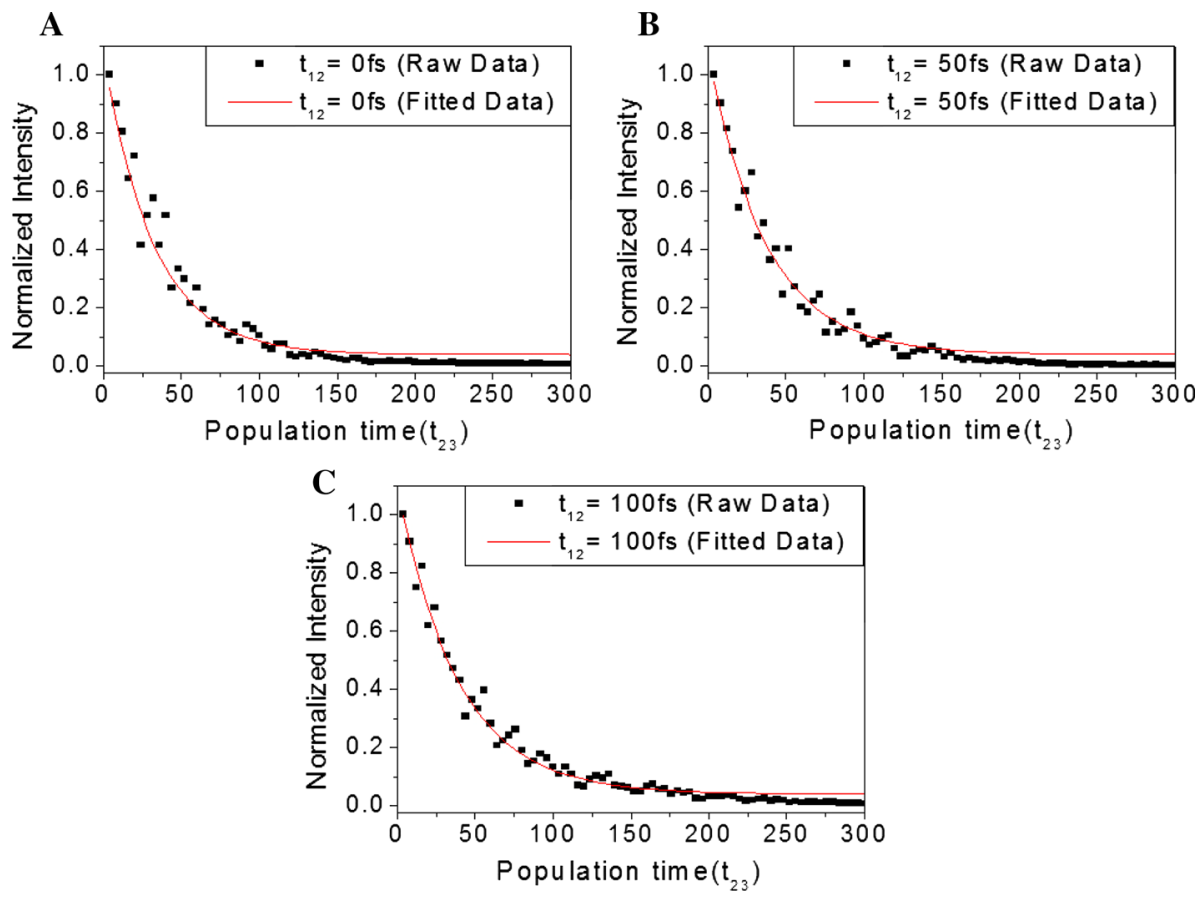

Figure 9. Fitted integrated plots of photon echo signal for CdSe 640 quantum dot in toluene solvent at four (A to D) specific coherence times $\left(\mathrm{t}_{12}\right)$ while varying the population $\left(\mathrm{t}_{23}\right)$. 
Table 1. Decay constants of CdSe 610 and CdSe 640 quantum dots in toluene

\begin{tabular}{|c|c|c|c|c|c|}
\hline \multicolumn{2}{|c|}{ Coherence time $\left(\mathrm{t}_{12}\right)$} & \multicolumn{2}{|c|}{ Decay constant $\left(\tau_{1}\right)$} & \multicolumn{2}{|c|}{ Decay constant $\left(\tau_{2}\right)$} \\
\hline CdSe 610 & CdSe 640 & CdSe 610 & CdSe 640 & CdSe 610 & CdSe 640 \\
\hline $\mathrm{t}_{12}=0 \mathrm{fs}$ & $\mathrm{t}_{12}=0 \mathrm{fs}$ & $32 \mathrm{fs}$ & $32 \mathrm{fs}$ & $1.13 \mathrm{ps}$ & $2.05 \mathrm{ps}$ \\
\hline $\mathrm{t}_{12}=50 \mathrm{fs}$ & $\mathrm{t}_{12}=50 \mathrm{fs}$ & $37 \mathrm{fs}$ & $37 \mathrm{fs}$ & $1.26 \mathrm{ps}$ & $2.17 \mathrm{ps}$ \\
\hline $\mathrm{t}_{12}=100 \mathrm{fs}$ & $\mathrm{t}_{12}=100 \mathrm{fs}$ & $39 \mathrm{fs}$ & $39 \mathrm{fs}$ & $1.13 \mathrm{ps}$ & $2.19 \mathrm{ps}$ \\
\hline
\end{tabular}

dots samples. The integrated plots of photon signal give valuable information about population relaxation time by varying the population time $\left(t_{23}\right)$ at different fixed coherence times $\left(\mathrm{t}_{12}\right)$. For collecting the time-resolved integrated signal, we have used $810 \mathrm{~nm}$ wavelength laser pulses, which are very far from the resonance condition for both quantum dots samples. Figure $8(\mathrm{~A}-\mathrm{C})$ shows the fitted integrated plots of photon echo signal for $\mathrm{CdSe}$ 610 quantum dot sample in toluene at $810 \mathrm{~nm}$ central wavelength of laser pulses.

In this case, we are scanning the population time $\left(\mathrm{t}_{23}\right)$ at different fixed coherence time 0 fs, 50 fs and 100 fs. The integrated photon echo signals are well fitted in the bi-exponential model and give two decay constants. At zero coherence time, two decay constants are $32 \mathrm{fs}$ and $1.13 \mathrm{ps}$ as given in Table 1 . The fast decay constant is pulse width limited, and the slow decay component shows intra-band relaxation dynamics. At 50 fs coherence time, two decay constants are $37 \mathrm{fs}$ and $1.26 \mathrm{ps}$. Finally, at 100 fs coherence time, the two decay constants are $39 \mathrm{fs}$ and $1.34 \mathrm{ps}$.

Figure $9(\mathrm{~A}-\mathrm{C})$ shows the fitted integrated plots of photon echo signal for CdSe 640 quantum dot. At zero delay or zero coherence time $\left(t_{12}\right)$, the two decay constants resulting from our bi-exponential model are $35 \mathrm{fs}$ and 2.05 ps respectively as shown in Figure 8(A), and values are given in Table 1.

We also measured the decay time scale by fitting the bi-exponential curve at different fixed coherence time $\left(t_{12}\right) 50$ and 100 as shown in Figure 9(B-C) and measured time scales are shown in Table 1.

\section{Conclusions}

In spite of experimenting with laser pulses centered at $810 \mathrm{~nm}$, a photon echo signal of substantial intensity is obtained. Thus, ultrafast dynamics can be studied using photon echo technique even under the far-fromresonance condition, which is an interesting observation, because all typical photon echo studies are done in the near-resonance condition, with the laser wavelength close to the maximum absorbance of the sample. In this situation, SRPE at far-resonance wavelength may still allow dynamical information to be extracted, provided there is no interference due to the solvent. A small difference is observed in the molecular dynamics of CdSe 610 and CdSe 640. The spectral diffusion process for CdSe 640 occurs within first 50 fs, while for CdSe 610, it may be occurring after 100 fs timescale. The fast decay constant for both CdSe 610 and CdSe 640 is 30-40 fs because of the limited pulse width. The slow decay constant for $\mathrm{CdSe} 610$ is in between 1.13 ps to $1.36 \mathrm{ps}$, while for CdSe 640, it is between 2.05 to 2.19 ps, at different fixed coherence times.

\section{Acknowledgements}

This work on the application of high nonlinear optical spectroscopy for understanding photochemistry is dedicated to my Teacher Prof. M. V. George. I thank several of my present and past graduate students for help with experiments. I also thank Mrs. S. Goswami for language editing. The laser system used in these experiments were made possible due to the DST-FIST (2nd Phase 2010) funds. Funding was provided by Indian Space Research Organisation (Grant No. STC) and Science and Engineering Research Board (Grant No. Intramural Research).

\section{References}

1. Jordanides X J, Lang M J, Song X and Fleming G R 1999 Solvation Dynamics in Protein Environments Studied by Photon Echo Spectroscopy J. Phys. Chem. B 1037995

2. Agarwal R, Pral B S, Rizvi A H, Yang M and Fleming G R 2002 Two-color three pulse photon echo peak shift spectroscopy J. Chem. Phys. 1166243

3. Mukamel S 1995 In Principles of nonlinear optical spectroscopy (New York: Oxford University Press)

4. Boyd R W 1992 In Nonlinear Optics (Boston: Academic Press)

5. Brixner T, Stenger J, Vaswani H M, Cho M, Blankenship R E and Fleming G R 2005 Two-dimensional spectroscopy of electronic couplings in photosynthesis Nature $\mathbf{4 3 4} 625$

6. Cho M, Vaswani H M, Brixner T, Stenger J and Fleming G R 2005 Exciton Analysis in 2D Electronic Spectroscopy J. Phys. Chem. B 10910542 
7. Engel G S, Calhoun T R, Read E L, Ahn T, Mančal T, Cheng Y, Blankenship R E, and Fleming G R 2007 Evidence for wavelike energy transfer through quantum coherence in photosynthetic systems Nature 446782

8. Shen Y R 1984 In Principles of Nonlinear Optics (New York: Wiley)

9. Dantus M 2001 Coherent Nonlinear Spectroscopy: From Femtosecond Dynamics to Control Ann. Rev. Phys. Chem. 52639

10. Fourkas J T 2002 Higher order optical correlation spectroscopy in liquids Annu. Rev. Phys. Chem. 5317

11. Dao L V, Lincoln C, Lowe M and Hannaford P 2003 Spectrally resolved two-colour three-pulse photon echo studies of vibrational dynamics of molecules Physica B: Condensed Matt. 327123

12. Dao L V, Lincoln C, Lowe M and Hannaford P 2004 Spectrally resolved femtosecond two-color three-pulse photon echoes: Study of ground and excited state dynamics in molecules J. Chem. Phys. 1208434

13. Lozovoy V V, Grimberg B I, Brown E J, Pastirk I and Dantus M 2000 Femtosecond spectrally dispersed threepulse four-wave mixing: the role of sequence and chirp in controlling intramolecular dynamics J. Raman Spec. 31 41

14. Demirdöven N, Khalil M, Golonzka O and Tokmakoff A 2001 Correlation Effects in the Two-Dimensional Vibrational Spectroscopy of Coupled Vibrations J. Phys. Chem. A 1058025

15. Volker S 1989 Hole-Burning Spectroscopy Annu. Rev. Phys. Chem. 40499

16. Joo T, Jia Y, Yu J, Lang M J and Fleming G R 1996 Third-order nonlinear time domain probes of solvation dynamics J. Chem. Phys. 1046089

17. De Boeij W P, Pshenichnikov M S and Wiersma D A 1996 System-Bath Correlation Function Probed by Conventional and Time-Gated Stimulated Photon Echo J. Phys. Chem. 10011806

18. Cho M, Yu J, Joo T, Nagasawa Y, Passino S A and Fleming G R 1996 The Integrated Photon Echo and Solvation Dynamics J. Phys. Chem. 10011944

19. De Boeij W P, Pshenichnikov M S, and Wiersma D A 1998 Ultrafast solvation dynamics explored by femtosecond photon echo spectroscopies Annu. Rev. Phys. Chem. 4999

20. Park J, and Joo T 2002 Nuclear dynamics in electronic ground and excited states probed by spectrally resolved four wave mixing J. Chem. Phys. 11610801

21. Book L D, and Scherer N F 1999 Wavelength-resolved stimulated photon echoes: Direct observation of ultrafast intramolecular vibrational contributions to electronic dephasing J. Chem. Phys. 111792

22. Karthick Kumar S, Tiwari V, Goswami T, and Goswami D 2009 Spectrally resolved photon echo spectroscopy of $\mathrm{Zn}$ (II), $\mathrm{Co}(\mathrm{II})$ and $\mathrm{Ni}$ (II)-octaethyl porphyrins Chem. Phys. Lett. 47631

23. Wehner M U, Steinbach D, and Wegener M 1996 Ultrafast coherent transients due to exciton-continuum scattering in bulk GaAs Phys. Rev. B 54 R5211

24. Hataoka S, Itoh A, Tanahashi I, and Tanaka K 2000 High-density excitation effects of excitons in $\mathrm{ZnSe}$ quantum wells by spectrally resolved four-wave mixing $J$. Lumin. 87-89 853
25. Klimov V I 2007 Spectral and Dynamical Properties of Multiexcitons in Semiconductor Nanocrystals Annu. Rev. Phys. Chem. $\mathbf{5 8} 635$

26. Scholes G D 2008 Controlling the Optical Properties of Inorganic Nanoparticles Adv. Funct. Mater. 181145

27. Kambhampati P 2011 Unraveling the Structure and Dynamics of Excitons in Semiconductor Quantum Dots Acc. Chem. Res. 441

28. Ekimov A I, Kudryavtsev I A, Efros A L, Yazeva T V, Hache F, Schanne-Klein M C, Rodina A V, Ricard D and Flytzanis C 1993 Absorption and intensity-dependent photoluminescence measurements on $\mathrm{CdSe}$ quantum dots: assignment of the first electronic transitions J. Opt. Soc. Am. B $\mathbf{1 0} 100$

29. Efros A L and Rosen M 2000 The Electronic Structure of Semiconductor Nanocrystals Annu. Rev. Mater. Sci. 30 475

30. Burda C, Chen X, Narayanan R and El-Sayed M A 2005 Chemistry and Properties of Nanocrystals of Different Shapes Chem. Rev. 1051025

31. Ekimov A, Efros A and Onushchenko A 1993 Quantum size effect in semiconductor microcrystals Solid State Comm. 88947

32. Henglein A 1982 Photo-Degradation and Fluorescence of Colloidal-Cadmium Sulfide in Aqueous Solution Ber. Bunsenges. Phys. Chem. 86301

33. Leatherdale C A, Woo W, Mikulec F V and Bawendi M G 2002 On the Absorption Cross Section of CdSe Nanocrystal Quantum Dots J. Phys. Chem. B 1067619

34. Murray C B, Norris D J and Bawendi M G 1993 Synthesis and characterization of nearly monodisperse $\mathrm{CdE}(\mathrm{E}$ $=$ sulfur, selenium, tellurium) semiconductor nanocrystallites J. Am. Chem. Soc. 1158706

35. Mohamed M B, Burda C and El-Sayed M A 2001 Shape Dependent Ultrafast Relaxation Dynamics of CdSe Nanocrystals: Nanorods vs Nanodots Nano Lett. 1 589

36. Norris D J and Bawendi M G 1996 Measurement and assignment of the size-dependent optical spectrum in CdSe quantum dots Phys. Rev. B 5316338

37. Soloviev V N, Eichhöfer A, Fenske D and Banin U 2000 Molecular Limit of a Bulk Semiconductor: Size Dependence of the "Band Gap" in CdSe Cluster Molecules $J$. Am. Chem. Soc. 1222673

38. Soloviev V, Eichhöfer A, Fenske D and Banin U 2001 Molecular Limit of a Bulk Semiconductor: Size Dependent Optical Spectroscopy Study of CdSe Cluster Molecules Phys. Stat. Sol. (b) 224285

39. Soloviev V N, Eichhöfer A, Fenske D and Banin U 2000 Molecular Limit of a Bulk Semiconductor: Size Dependence of the "Band Gap" in CdSe Cluster Molecules $J$. Am. Chem. Soc. 1222673

40. Nirmal M, Murray C B and Bawendi M G 1994 Fluorescence-line narrowing in CdSe quantum dots: Surface localization of the photogenerated exciton Phys. Rev. B $\mathbf{5 0} 2293$

41. Norris D J, Sacra A, Murray C B and Bawendi M G 1994 Measurement of the size dependent hole spectrum in CdSe quantum dots Phys. Rev. Lett. 72 2612

42. Sewall S L, Cooney R R and Kambhampati P 2009 Experimental tests of effective mass andatomistic 
approaches to quantum dot electronic structure: Ordering of electronic states Appl. Phys. Lett. 94243116

43. Guyot-Sionnest P, Shim M, Matranga C and Hines M 1999 Intraband relaxation in CdSe quantum dots Phys. Rev. B 60 R2181

44. Burda C, Link S, Mohamed M and El-Sayed M 2001 The Relaxation Pathways of CdSe Nanoparticles Monitored with Femtosecond Time-Resolution from the Visible to the IR: Assignment of the Transient Features by Carrier Quenching J. Phys. Chem. B 10512286

45. McArthur E A, Morris-Cohen A J, Knowles K E and Weiss E A 2010 Charge Carrier Resolved Relaxation of the First Excitonic State in CdSe Quantum Dots Probed with Near-Infrared Transient Absorption Spectroscopy J. Phys. Chem. B 11414514

46. Norris D J and Bawendi M G 1995 Structure in the lowest absorption feature of CdSe quantum dots J. Chem. Phys. 1035260

47. Klimov V I 2000 Optical Nonlinearities and Ultrafast Carrier Dynamics in Semiconductor Nanocrystals $J$. Phys. Chem. B 1046112

48. Lupo M G, Della Sala F, Carbone L, Zavelani-Rossi M, Fiore A, Lüer L, Polli D, Cingolani R, Manna L and Lanzani G 2008 Ultrafast Electron-Hole Dynamics in Core/Shell CdSe/CdS Dot/Rod Nanocrystals Nano Lett. 84582

49. Yu P, Nedeljkovic J M, Ahrenkiel P A, Ellingson R J and Nozik A J 2004 Size Dependent Femtosecond Electron Cooling Dynamics in CdSe Quantum Rods Nano Lett. 4 1089

50. He J, Zhong $\mathrm{H}$ and Scholes G D 2010 ElectronHole Overlap Dictates the Hole Spin Relaxation Rate in Nanocrystal Heterostructures Phys. Rev. Lett. 105 046601

51. Scholes G D, Kim J, Wong C Y, Huxter V M, Nair P S, Fritz K P and Kumar S 2006 Nanocrystal Shape and the Mechanism of Exciton Spin Relaxation Nano Lett. 6 1765

52. Graham M W, Ma Y and Fleming G R 2008 Femtosecond Photon Echo Spectroscopy of Semiconducting SingleWalled Carbon Nanotubes Nano Lett. 83936

53. Salvador M R, Sreekumari Nair P, Cho M and Scholes G D 2008 Interaction between excitons determines the non-linear response of nanocrystals Chem. Phys. 35056

54. Wong C Y and Scholes G D 2011 Biexcitonic Fine Structure of CdSe Nanocrystals Probed by Polarization-Dependent Two-Dimensional Photon Echo Spectroscopy J. Phys. Chem. A 1153797

55. Turner D B, Hassan Y and Scholes G D 2012 Exciton Superposition States in CdSe Nanocrystals Measured Using Broadband Two-Dimensional Electronic Spectroscopy Nano Lett. 12880

56. Nagasawa Y, Watanabe A, Takikawa $\mathrm{H}$ and Okada T 2003 Solute Dependence of Three Pulse Photon Echo Peak Shift Measurements in Methanol Solution J. Phys. Chem. A 107632

57. Xu Q, Scholes G D, Yang M and Fleming G R 1999 Probing Solvation and Reaction Coordinates of Ultrafast Photoinduced Electron-Transfer Reactions Using Nonlinear Spectroscopies: Rhodamine 6G in ElectronDonating Solvents J. Phys. Chem. A 10310348
58. Bürsing H, Ouw D, Kundu S and Vöhringer P 2001 Probing solvation dynamics in liquid water and at phospholipid/water interfaces with femtosecond photon-echo spectroscopies Phys. Chem. Chem. Phys. 32378

59. Parkinson D Y, Lee H and Fleming G R 2007 Measuring Electronic Coupling in the Reaction Center of Purple Photosynthetic Bacteria by Two-Color, ThreePulse Photon Echo Peak Shift Spectroscopy J. Phys. Chem. B 1117449

60. Salvador M R, Graham M W and Scholes G D 2006 Exciton-phonon coupling and disorder in the excited states of CdSe colloidal quantum dots J. Chem. Phys. 125 184709

61. Zhang W M, Meier T, Chernyak V and Mukamel S 1998 Exciton-migration and three-pulse femtosecond optical spectroscopies of photosynthetic antenna complexes $J$. Chem. Phys. 1087763

62. Jimenez R, Van Mourik F, Yu J Y and Fleming G R 1997 Three-Pulse Photon Echo Measurements on LH1 and LH2 Complexes of Rhodobacter sphaeroides: A Nonlinear Spectroscopic Probe of Energy Transfer J. Phys. Chem. B 1017350

63. Meier T, Chernyak V and Mukamel S 1997 Femtosecond photon echoes in molecular aggregates J. Chem. Phys. 1078759

64. McKimmie L J, Lincoln C N, Jasieniak J and Smith T A 2009 Three-Pulse Photon Echo Peak Shift Measurements of Capped CdSe Quantum Dots J. Phys. Chem. C 11482

65. Gong S, Yao D, Jiang H and Xiao H 2008 Parameterdependent photon echo induced in $\mathrm{CdSe} / \mathrm{ZnS}$ quantum dot quantum well Phys. Lett. A 3723325

66. Colonna A E, Yang X and Scholes G D 2005 Photon echo studies of biexcitons and coherences in colloidal CdSe quantum dots Phys. Stat. Sol. (b) 242990

67. Mittleman D M, Schoenlein R W, Shiang J J, Colvin V L, Alivisatos A P and Shank C V 1994 Quantum size dependence of femtosecond electronic dephasing and vibrational dynamics in CdSe nanocrystals Phys. Rev. B 4914435

68. Takemoto K, Ikezawa M and Masumoto Y 2003 Lowtemperature dephasing mechanism of very small quantum dots: the role of confined phonons and surrounding matrices Phys. Stat. Sol. (c) 01279

69. Masumoto Y, Ikezawa M, Hyun B, Takemoto K and Furuya M 2001 Homogeneous Width of Confined Excitons in Quantum Dots at Very Low Temperatures Phys. Stat. Sol. (b) 224613

70. Scholes G D 2004 Selection rules for probing biexcitons and electron spin transitions in isotropic quantum dot ensembles J. Chem. Phys. 12110104

71. Mukamel S, Piryatinski A and Chernyak V 1999 TwoDimensional Raman Echoes: Femtosecond View of Molecular Structure and Vibrational Coherence Acc. Chem. Res. 32145

72. Hannaford P 2005 In Femtosecond laser spectroscopy (New York: Springer)

73. Aue W P, Bartholdi E and Ernst R R 1976 Twodimensional spectroscopy. Application to nuclear magnetic resonance J. Chem. Phys. 642229

74. Mukamel S 1995 In Principles of Nonlinear Optical Spectroscopy (New York: Oxford University Press) 
75. Alivisatos A P, Harris A L, Levinos N J, Steigerwald M L and Brus L E 1988 Electronic states of semiconductor clusters: Homogeneous and inhomogeneous broadening of the optical spectrum J. Chem. Phys. 894001

76. Klimov V I, McBranch D W, Leatherdale C A and Bawendi M G 1999 Electron and hole relaxation pathways in semiconductor quantum dots Phys. Rev. B 60 13740

77. Salvador M R, Hines M A and Scholes G D 2003 Exciton-bath coupling and inhomogeneous broadening in the optical spectroscopy of semiconductor quantum dots J. Chem. Phys. 1189380

78. Klimov V, Bolivar P H and Kurz H 1996 Ultrafast carrier dynamics in semiconductor quantum dots Phys. Rev. $B \mathbf{5 3} 1463$
79. Efros A L, Kharchenko V and Rosen M 1995 Breaking the phonon bottleneck in nanometer quantum dots: Role of Auger-like processes Solid State Commun. 93281

80. Thomas D and Michael F 2007 In Coherent Vibrational Dynamics (New York: CRC Press)

81. Sagar D M, Cooney R R, Sewall S L, Dias E A, Barsan M M, Butler I S and Kambhampati P 2008 Size dependent, state-resolved studies of exciton-phonon couplings in strongly confined semiconductor quantum dots Phys. Rev. B 77235321

82. Klimov V I and McBranch D W 1998 Femtosecond 1P-to-1S Electron Relaxation in Strongly Confined Semiconductor Nanocrystals Phys. Rev. Lett. 80 4028 